\title{
Gut microbiome diversity in acute infective and chronic inflammatory gastrointestinal diseases in North India
}

\author{
Saurabh Kedia ${ }^{1} \cdot$ Ritika Rampal $^{1} \cdot$ Jaishree Paul $^{2} \cdot$ Vineet Ahuja $^{1}$
}

Received: 12 February 2016/ Accepted: 23 February 2016/Published online: 19 March 2016

(C) Japanese Society of Gastroenterology 2016

\begin{abstract}
The disease profile in the Indian population provides a unique opportunity for studying the host microbiome interaction in both infectious (amebiasis) and autoimmune diseases like inflammatory bowel disease (IBD) from a similar environment and genetic background. Analysis of fecal samples from untreated amebic liver abscess (ALA) patients, Entamoeba histolytica (Eh)-negative and -positive asymptomatic individuals, and pus samples from naive ALA patients revealed a significant reduction in Lactobacillus in asymptomatic individuals (Eh $+\mathrm{ve})$ and ALA patients. Two anaerobic genera, namely Bacteroides and Peptostreptococcus, were detected in naive ALA pus samples. Analysis of fecal samples from amoebic colitis patients showed a significant decline in population of Bacteroides, Clostridium coccoides and leptum subgroup, Lactobacillus, Campylobacter, and Eubacterium, whereas a significant increase in Bifidobacterium was observed. Mucosa-associated bacterial flora analysis from IBD patients and healthy controls revealed a significant difference in concentration of bacteria among predominating and subdominating genera between ulcerative colitis (UC), Crohn's disease (CD) patients, and controls. In contrast to the mucosal studies, we found a significant increase in lactobacilli population in fecal samples of active UC patients. Another study revealed a significant decrease of Clostridium coccoides and leptum clusters in fecal samples of active UC patients along with
\end{abstract}

Vineet Ahuja

vineet.aiims@gmail.com

1 Department of Gastroenterology, All India Institute of Medical Sciences, New Delhi, India

2 School of Life Sciences, Jawaharlal Nehru University, New Delhi, India decreased concentrations of fecal SCFAs, especially of $n$ butyrate, iso-butyrate, and acetate. We therefore found similar perturbations in gut microbiome in both infectious and autoimmune diseases, indicating inflammation to be the major driver for changes in gut microbiome.

Keywords Gut microbiome - Amebiasis - Amoebic liver abscess · Ulcerative colitis · Crohn's disease

\section{Introduction}

The human gut harbors more than $10^{14}$ microorganisms, comprising more than 500-1000 species [1-3]. These include bacterial, microeukaryotic, and viral populations [4]. The human gastrointestinal tract and these microbiota form a unique ecosystem, and this association of host and microbiome contributes to both health and disease status of the host. The interaction between host and gut microbiome starts right from birth and continues throughout the life. The microbiota colonize the gut soon after birth and the mother of the host is the first source for these gut microbiota [5, 6]. A number of factors, including diet [7-9], age [10], gender, genetic composition [11], geographic location [2], and health/disease status [6] of the individual influence the gut microbiota after birth. The microbiota varies in their diversity and stability with advancing age. In early life, the microbiome is characterized by low diversity and low stability [12] and the microbiome gains stability and diversity by early adulthood [13, 14] (Fig. 1). Metagenome refers to the genetic contents of entire microbial community residing in the human gut. Metagenome of the human gut colonizers has more than 100 times the number of genes as compared to human genome [15]. The greatest population density of these microbes is observed in distal 
Fig. 1 Factors affecting gut microbial diversity and stability
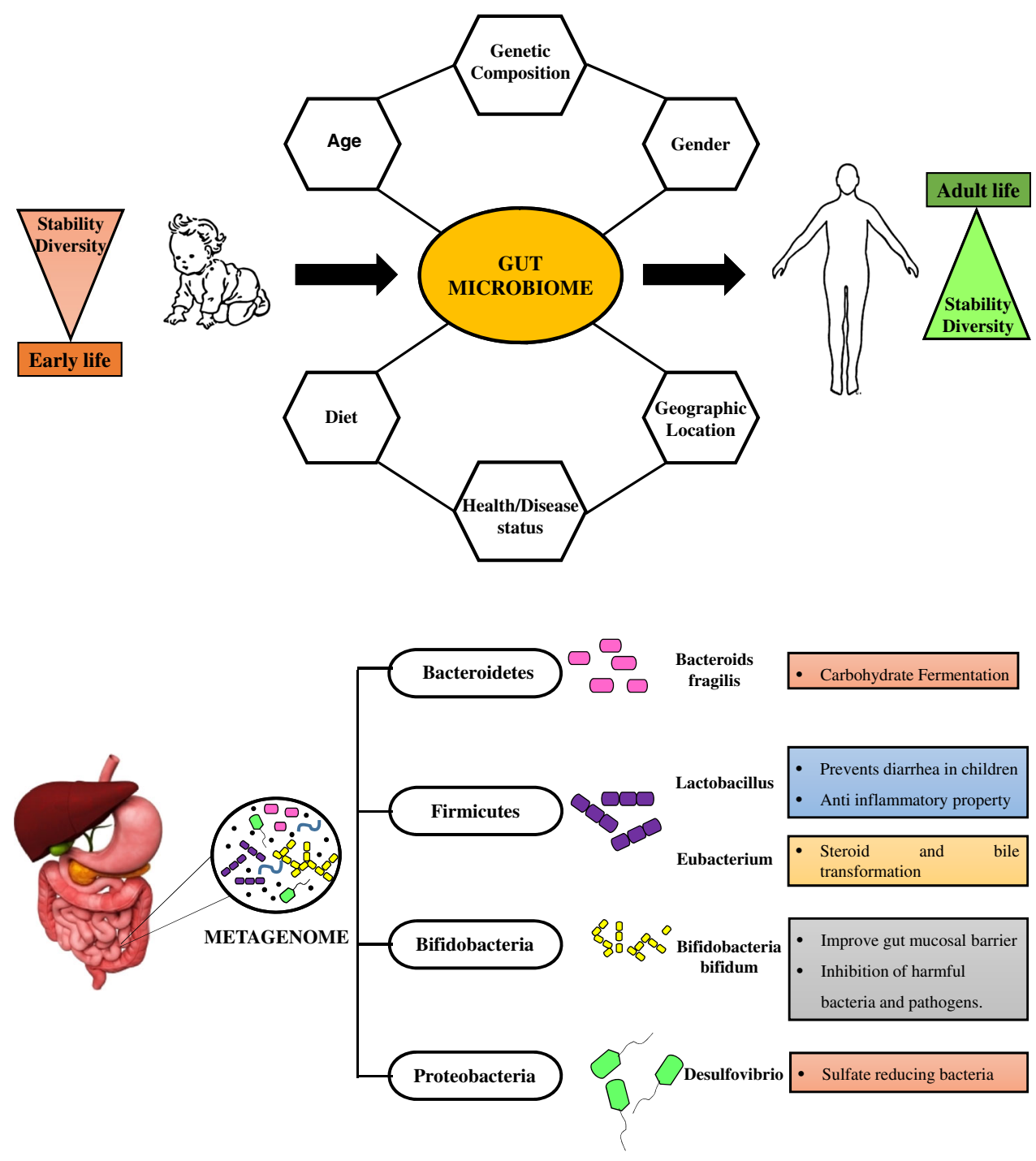

Fig. 2 Major bacterial constituents of gut metagenome ileum and colon [1]. The predominant microbial flora of human GI tract includes the following phyla: Bacteroidetes, Firmicutes, Proteobacteria (includes Enterobacteriaceae), Actinobacteria, Fusobacteria, and Verrucomicrobium (Fig. 2). Of these, Firmicutes and Bacteroides form more than $90 \%$ of the bacterial population present in the colon [16].

- Bacteroides species of the Bacteroidetes phylum are the most dominant species, and are predominant starch degraders. They play a very important role in carbohydrate metabolism, nutrition, and maintenance of health [17].

- Eubacterium genus of the Firmicutes phylum is the second most dominant species. The members of the genus Eubacterium are known to produce butyrate [18], degrade flavonoids (from vegetables, fruits, nuts, and tea) [19], and are implicated in steroid and bile transformation in intestine. Other members include
Clostridium group IV and XIVa, which are predominant butyrate-producing strains [20]. Non-butyrateproducing members include Ruminococcus torques and $R$. gnavus, which are among the primary mucindegrading organisms [21]. The genus Lactobacillus, comprising a large heterogenous group of low $\mathrm{G}+\mathrm{C}$ Gram-positive, non-sporulating, anaerobic bacteria and are other important members of the phylum Firmicutes. Lactobacilli are known to fortify the epithelial barrier by various mechanisms such as induction of mucin secretion, enhancement of tight-junction functioning, upregulation of cytoprotective heat shock proteins, and prevention of apoptosis of epithelial cells [22]. Probiotic strains of Lactobacillus are known to prevent infectious diarrhea, antibiotic-associated diarrhea, and diarrhea in children who are unusually more susceptible as a result of poor nutrition, impaired immune status, or frequent exposure to pathogens [23]. 
- Members of Bifidobacteria (member of Actinobacteria phylum) produce acetate in proximal and distal colon by fermentation of glucose and fructose [24]. Members of both Bifidobacteria and Ruminococcus (Ruminococcus torques and Bifidobacterium bifidum) are thought to ferment mucin and compete to colonize this substrate for their energy source [25].

- Sulfate-reducing bacteria are found in five distinct genera in the delta subdivision of the Proteobacteria phylum [26]. Hydrogen-consuming, sulfate-reducing bacteria are found in two of these genera, e.g., Desulfovibrio [27].

Of the above-mentioned bacteria, Bacteroides, Bifidobacterium, Eubacterium, Clostridium, Peptococcus, Peptostreptococcus, Lactobacillus, and Ruminococcus are considered to be predominant genera, whereas Enterococcus, Methanobrevibacter, and sulphur-reducing bacteria (SRB) remain as the subdominant genera [28]. Both the host and gut microbiota benefit each other as microbes benefit from the nutrient-rich host GI tract and the host benefits from the metabolic abilities of the microbes. The beneficial effects of these microbiota (which form a 'microbial organ' within the intestine) [29] are mentioned in the Fig. 3.

Gut microbiome in both health and disease are currently under intense investigation worldwide by scientists and clinicians with diverse expertise and interests. We present our experience of interrogating gut microbiome, looking at changes in its diversity and composition in gut parasitic diseases like amebiasis, which are very prevalent in tropical countries and in organ-specific auto-immune diseases like inflammatory bowel disease (IBD), which is rapidly emerging in the tropical countries.

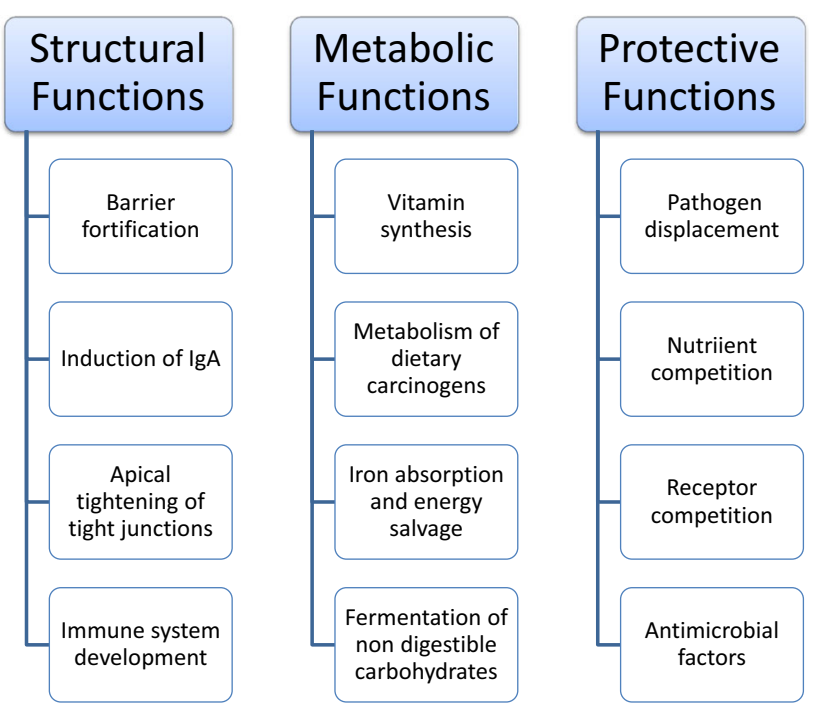

Fig. 3 Gut microbiome functions in human health

\section{Intestinal flora and amebiasis}

Entamoeba histolytica is a micro-aerophilic protozoan that is the causative agent of invasive amebiasis, including colitis and liver abscess. It is still a significant cause of morbidity and mortality in developing countries like India [30]. Although both the protozoan and intestinal flora reside together in the gut, their interaction and the contribution of gut flora towards the manifestation of invasive amebiasis is not well understood. Amebiasis is initiated with ingestion of $E$. histolytica cysts through fecally contaminated water/food. Trophozoites are released after excystation of these cysts in the intestinal lumen. After infection in most of the individuals, these trophozoites usually live as commensals without causing any damage to the host, and very few individuals develop invasive disease [31]. India is endemic for amebiasis, and the total number of infected individuals is very high (up to $20 \%$ of the Indian population). These trophozoites initiate invasive disease when they penetrate the mucus layer and damage the intestinal tissues $[32,33]$. The trophozoites drive their nutrition by ingesting intestinal flora [34]. The bacterial flora also provides anaerobic conditions or low redox potential, which is beneficial for amoebic growth [35]. However, the amoeba are very selective in their interaction with the different bacterial species, and only those bacteria that have appropriate receptors are ingested by these trophozoites [36]. It has also been proposed that intestinal flora may also influence the virulence potential of Entamoeba, as certain bacterial species may trigger virulence while others my decrease it [37]. Fluctuations in gut flora have been reported in acute diarrhea and antibiotic-associated diarrhea [33]. However, little was known about the Entamoeba-gut flora cross talk. Therefore, our group studied the clinical correlation between resident bacterial flora and severity and spectrum of amoebic disease.

In a collaborative study [38], we looked into the profile of predominant gut flora of healthy individuals, asymptomatic E. histolytica carriers, and amebic liver abscess patients. We also addressed the issue of whether the changes observed in the gut flora may be attributed to the administration of metronidazole or the presence of the parasite. We also looked for the presence of gut bacteria in the liver abscess aspirates. The prevalence of the metronidazole-resistance gene ( $\mathrm{nim}$ ) was also scored in these bacterial species. Stool samples were analyzed from the following categories of individuals: (1) healthy $E$. histolytica-negative (2) healthy, asymptomatic, E. histolyticapositive, and (3) amoebic liver abscess (ALA) patients. Pus samples were also analyzed from ALA patients for the presence of 11 prominent, mostly anaerobic, bacteria found in the human gut. Bacterial detection was done by PCR 
Fig. 4 Dysbiosis in Entamoeba histolytica colitis as compared to healthy individuals

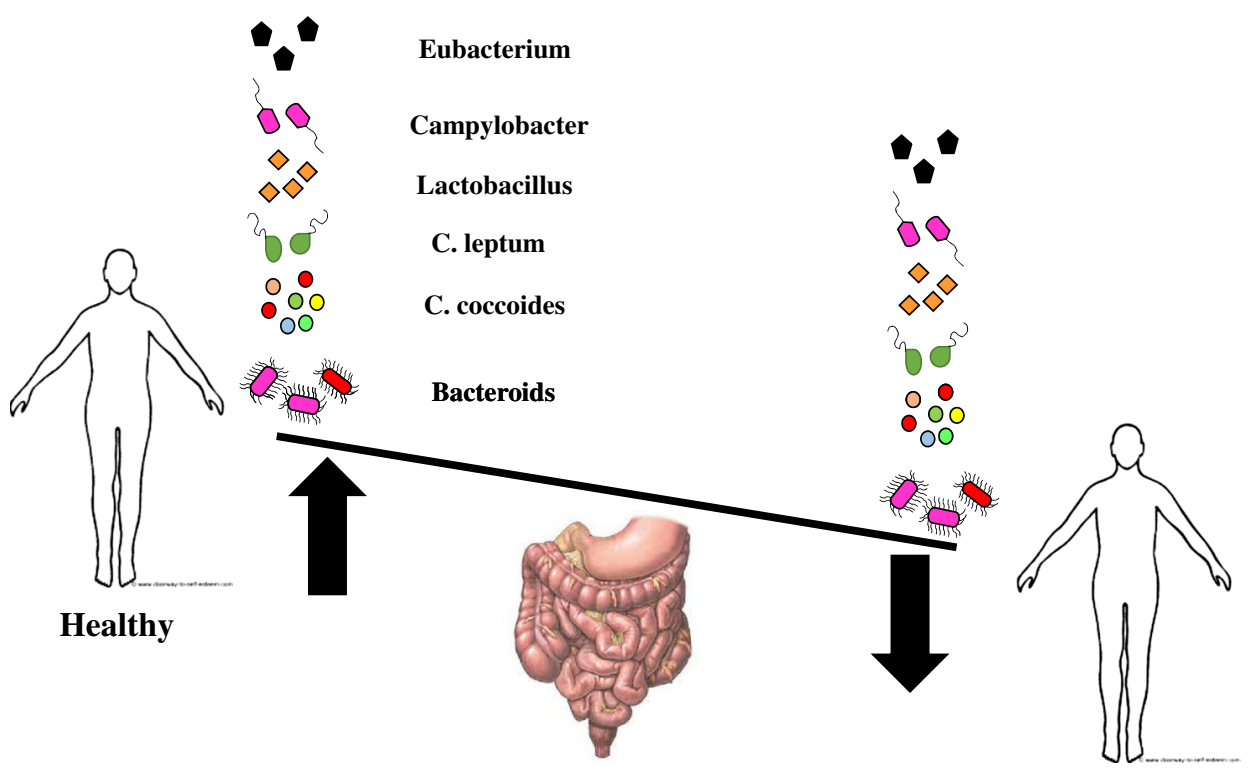

E. histolytica + amplification of total DNA from samples, using genusspecific primers for the $16 \mathrm{~S}$ rRNA gene. The specificity of detection was ascertained by sequencing the PCR-amplified bands and confirming their identity by comparing with genus-specific sequences available in the database. There was a statistically significant drop in the frequency of four of the 11 bacterial genera, namely, Bacteroides, Bifidobacterium, Lactobacillus, and Clostridium in fecal samples of ALA patients when compared with healthy, $E$. histolytica-negative controls (Fig. 4). Of these, three genera (Bacteroides, Bifidobacterium, and Clostridium) were not reduced in healthy asymptomatic carriers $(E$. histolytica-positive) compared with healthy $E$. histolytica-negative individuals. However, Lactobacillus was reduced in $E$. histolytica-asymptomatic carriers also. Asymptomatic carriers also showed a statistically significant decrease in the incidence of E. coli and increase in Pseudomonas aeruginosa compared with the other two categories of individuals. No significant changes were observed for Peptostreptococcus productus, Ruminococcus, Campylobacter, and Peptococcus among healthy controls and ALA patients (Table 1).

Patients with ALA were on metronidazole for ethical reasons while $E$. histolytica-positive asymptomatic carriers did not receive metronidazole. This could account for the observed reduction in Bacteroides, Bifidobacterium, and Clostridium in ALA patients alone [39]. On the other hand, Lactobacillus was reduced in both ALA patients and asymptomatic E. histolytica carriers possibly due to Entamoeba infection.

We confirmed the effect of metronidazole on intestinal flora by testing healthy volunteers (residents of an urban area) and irritable bowel syndrome (IBS) patients before and after taking metronidazole (Table 2). We observed

Table 1 Prevalence of selected bacteria in fecal samples from healthy individuals and ALA patients

\begin{tabular}{|c|c|c|c|c|}
\hline Bacterial species & $\begin{array}{l}\text { E. histolytica negative } \\
(n=19)\end{array}$ & E. histolytica positive $(n=11)$ & $\begin{array}{l}\text { Patients with amoebic liver } \\
\text { abscess }(n=19)\end{array}$ & $\begin{array}{l}\text { Significant } \\
p \text { value }\end{array}$ \\
\hline Bifidobacteria & 16 & 8 & 4 & $\mathrm{Gr}^{\#}$ I vs. Gr III \\
\hline Clostridium & 14 & 8 & 4 & Gr I vs. Gr III \\
\hline Ruminococcus & 17 & 7 & 12 & \\
\hline Campylobacter & 7 & 7 & 10 & \\
\hline Pseudomonas & 6 & 10 & 7 & \\
\hline Lactobacillus & 18 & 4 & 3 & Gr I vs. Gr 2 vs. Gr 3 \\
\hline Peptococcus & 12 & 9 & 8 & \\
\hline Bacteroides & 12 & 9 & 3 & Gr I vs. Gr III \\
\hline Peptostreptococcus & 12 & 10 & 14 & \\
\hline
\end{tabular}

Gr${ }^{\#}$ : Group, Gr I E. histolytica negative patients, Gr II E. histolytica positive patients, Gr III: Patients with amoebic liver abscess 
Table 2 Effect of metronidazole on the prevalence of selected bacteria in fecal samples in healthy Individuals

\begin{tabular}{lccl}
\hline Bacterial species & Pre metronidazole $(n=11)$ & Post metronidazole $(n=11)$ & $p$ value \\
\hline Bifidobacteria & 11 & 4 & 0.0039 \\
Clostridium & 11 & 5 & 0.0124 \\
Ruminococcus & 10 & 5 & $\mathrm{~ns}$ \\
Campylobacter & 9 & 1 & 0.0019 \\
Pseudomonas & 7 & 4 & $\mathrm{~ns}$ \\
Lactobacillus & 11 & 11 & $\mathrm{~ns}$ \\
Peptococcus & 11 & 6 & 0.035 \\
Bacteroides & 11 & 2 & 0.002 \\
Peptostreptococcus & 11 & 8 & $\mathrm{~ns}$ \\
\hline
\end{tabular}

similar results and found that there was a marked drop in the number of Bacteroides, Bifidobacterium, and Clostridium, while there was no effect on Lactobacillus. This could be explained by anaerobic spectrum of metronidazole. Bacteroides, Bifidobacterium, and Clostridium being obligate anaerobes are reduced by metronidazole treatment, whereas Lactobacillus being facultative anaerobe is not affected.

The second remarkable observation from this study was the highly significant occurrence of Peptostreptococcus (25/35 ALA cases, $71.4 \%$ occurrence) and, less frequently, Bacteroides (5/35 ALA cases, $14.2 \%$ occurrence) in the pus samples of ALA patients. The possible mechanisms for the presence of these bacteria in the pus samples could be intestinal bacterial overgrowth, increased permeability of mucosal barrier, and deficiency in host immune response.

Metronidazole resistance-associated genes (nim genes) have been associated with anaerobes including Bacteroides and Peptostreptococcus [40, 41]. We determined the presence of nim genes [42, 43] in all fecal and pus samples by PCR amplification with nim-specific primers. Amplicons of the sizes expected for the nim gene were present in both pus and fecal samples of ALA patients. However, these were not observed in E. histolytica-negative individuals and asymptomatic carriers. Resistance genes were present only in ALA patients because of rapid amplification of these genes in bacteria after metronidazole exposure. This was further confirmed by demonstrating these genes in healthy volunteers and IBS patients after metronidazole exposure.

In another study, real-time PCR (RT-PCR) was used for absolute quantification of gut flora in patients suffering from amoebic dysentery for 5-7 days. We also studied the presence of nim genes in this group of patients. We observed a significant decrease in population of $\mathrm{Bac}$ teroides, Clostridium coccoides subgroup, Clostridium leptum subgroup, Lactobacillus, Campylobacter, and Eubacterium in E. histolytica-positive samples when compared to that of healthy control samples (Fig. 3). Surprisingly, we observed a significant rise in the population of Bifidobacterium in amebic samples. There was no change in the population of Ruminococcus. This paradox can be explained by competition of Bifidobacterium and Ruminococcus for mucus as their energy source. Mucus secretion is increased in amebiasis as a result of the mechanism exerted by intestinal epithelial cells to counter the adherence of E. histolytica trophozoites to intestinal epithelial surface. This promotes increased colonization of Bifidobacteria. Bifidobacteria are also known to protect the gut through production of acetate. We also observed a significant increase in copy of nim gene in E. histolyticapositive samples compared to samples from healthy persons.

Such changes in bacterial population in the normal microbiota could have considerable consequences in terms of functional potential of gut flora and could result in metabolic conditions favorable for the establishment of opportunistic pathogens (e.g., Clostridium difficile). However, our study cannot conclude that observed changes in the gut flora are the cause or effect of the infection or the effect of dysenteric mechanism per se by the parasite. These findings have laid the platform for further research into dietary/probiotic interventions, which would have an impact on the gut flora-Entamoeba interaction for the benefit of the host.

\section{Gut flora and inflammatory bowel disease}

IBD is believed to result from an abnormal immune response to intestinal microbiota in genetically susceptible individuals [44]. Indirect evidence for the role of microbiota in the pathogenesis of IBD includes studies that have demonstrated evidence of mucosal $\mathrm{T}$ cells against gut microbiota [45] and mucosal secretion of immunoglobulin $\mathrm{G}$ antibodies in IBD patients [46] (Fig. 5). Recent trials in mild-to-moderate Crohn's disease (CD) have shown improvement with rifaximin [47].

Gut microbiome studies in IBD patients have demonstrated reduced diversity in the bacterial population [48], 


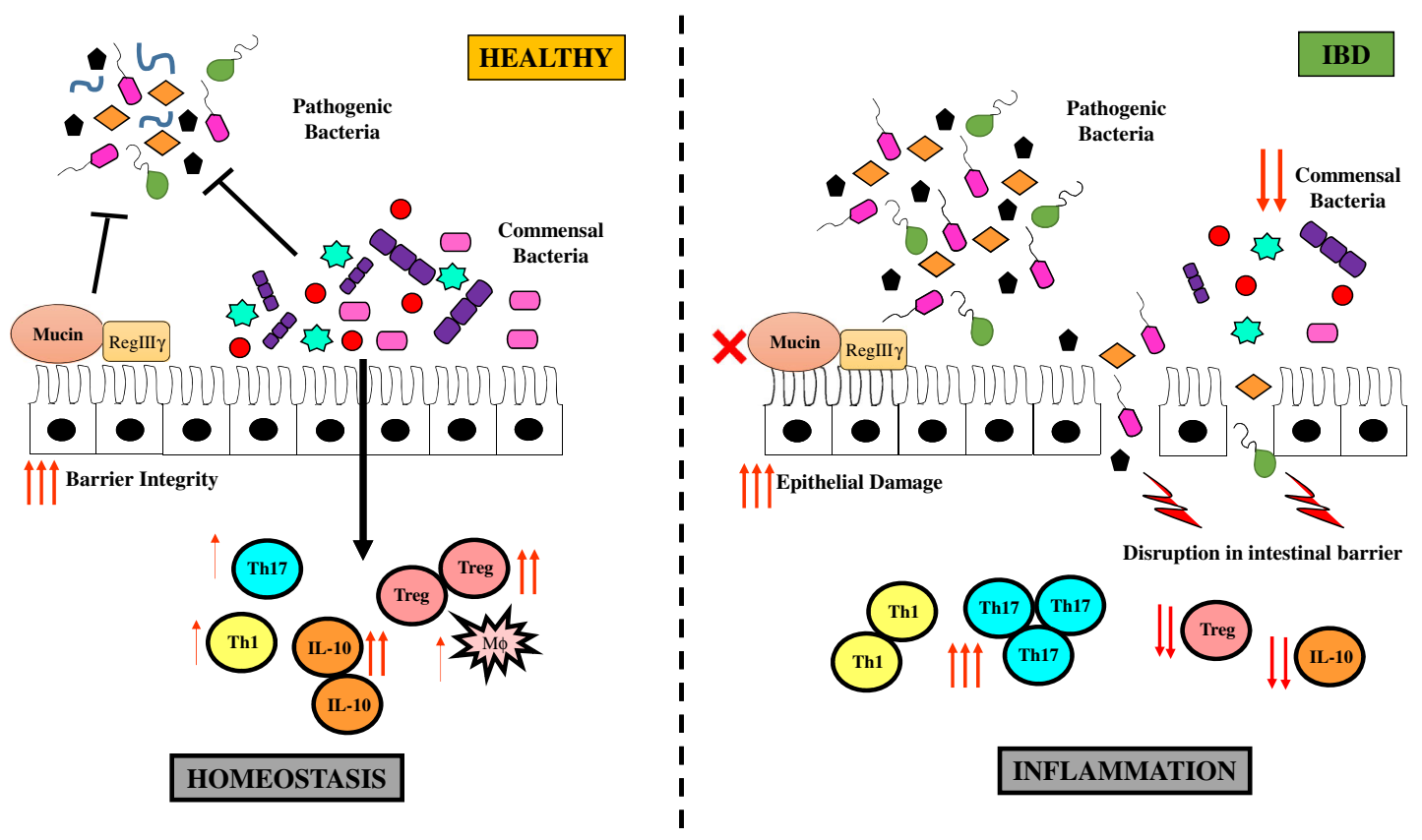

Fig. 5 Interplay of microbiome and immune system in healthy gut and in chronically inflamed gut

especially because of reduced diversity within the Firmicutes phylum [49-51]. Few studies have also shown an increased diversity in fungal population in patients with IBD [52, 53].

Studies from IBD patients and mouse models have shown increased population of pathogenetic bacteria including Enterobacteriaceae, especially Escherichia coli (adherent-invasive strains) [54]. These bacteria have been isolated from ileal biopsy samples in ileal CD [55] and ulcerative colitis (UC) patients [56]. Treatment with mesalamine has shown to decrease the population of these bacteria. Fusobacterium is another group of pathogenetic adherent-invasive bacteria, which have been found to be increased in colonic mucosa of UC patients [57, 58]. A number of protective bacterial species have been found to be decreased in IBD patients. These include Bifidobacterium, Lactobacillus [59], Faecalibacterium [60]. Lower levels of Faecalibacterium prausnitzii have been associated with a higher risk of disease recurrence after surgery in Crohn's disease patients [61].

We studied mucosa-associated bacterial flora from control individuals and IBD patients by real-time analysis using 16S rRNA-based genus-specific primers [62] (Fig. 6). There was a significant decline in the population of Bacteroides, Lactobacillus, Ruminococcus, and Bifidobacterium bacteria in both ulcerative colitis (UC) and Crohn's disease (CD) patients. Similar results have been reported in earlier studies [63-65]. The Clostridium leptum subgroup was reduced non-significantly in CD patients but increased sharply in UC patients, as observed in earlier studies [65-67]. The difference was more pronounced between UC and $\mathrm{CD}$ patients. The $C$. leptum group encompasses several butyrate-producing bacterial strains; hence, the decreases in their populations could play a role in the onset of CD. On the other hand, coccoid rods of Gram-positive Eubacterium and Peptostreptococcus bacteria were increased significantly in CD patients but not in UC patients. Campylobacter bacteria were significantly increased in both UC and CD patients when compared with the levels for the controls, and the levels for UC and CD patients were significantly different from each other. With the severity of the disease, the Campylobacter population increased significantly but reverted to normal during the remission stage. Our study also recorded increases in the populations of two subdominant inhabitants, the methanogenic bacterium Methanobrevibacter and sulfate-reducing bacteria (SRB) in both UC and CD patients, when compared with the levels for the controls. Therefore, there is clear delineation in concentration of bacteria between the predominating and subdominating genera under disease conditions, indicating that the subsets of bacteria participating in the pathogenesis of UC and CD are likely to be different [68].

In contrast to the mucosal studies, we found that lactobacilli in the fecal samples in patients with active UC are significantly increased as compared to healthy controls (unpublished data). Validation of Lactobacillus genusspecific probe was done by FISH-microscopy where hybridization of the probe was clearly seen with members of Lactobacillus/Enterococcus group. These levels of 


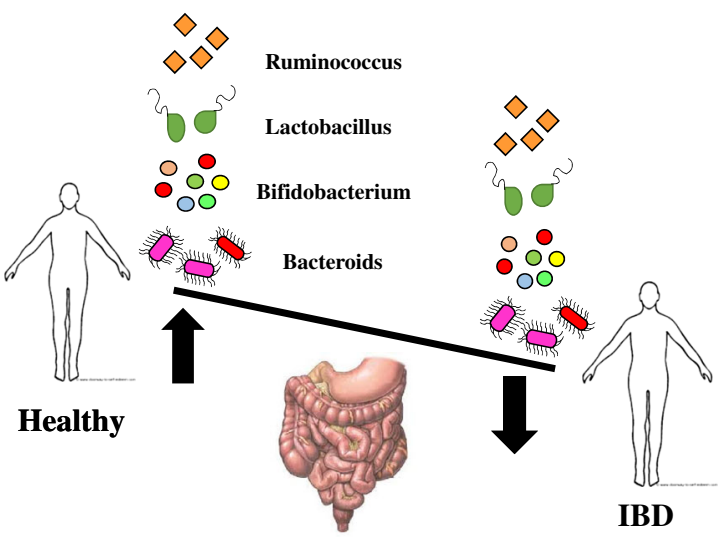

Fig. 6 Dysbiosis in IBD as compared to healthy individuals

Lactobacilli significantly reverted back to normal during remission, close to controls. Similar results have also been reported in other studies [69-71]. This was further supported by an increase in fecal lactate level (as measured by gas chromatography) in severe UC patients as compared to controls. Increased lactate level in IBD fecal samples has been detected earlier [72] and has been found to be associated with a higher risk of diarrhea and mucosal inflammation [73]. To explain the abundance of lactobacilli in fecal samples, we found significantly decreased expression of MUC9 mRNA in colonic mucosa of active UC patients as compared to controls. Therefore, increased fecal Lactobacilli population during active condition could be due to loss of mucin necessary for their adherence to the intestinal epithelial cell lining $[74,75]$. We also found a high degree of diversity in moderate and severe category of samples [by urea PAGE (polyacrylamide gel electrophoresis) fingerprinting] as compared to controls.

In addition to differences at the phylogenetic level, there are differences in the functional composition of gut microbiota in IBD patients as compared to controls. One of the most important functions is production of short-chain fatty acids (SCFAs) by fermentation of undigested carbohydrates. These SCFAs have been depicted to regulate transepithelial transport [76], colonocyte proliferation and differentiation [77], mucosal inflammation [78, 79], intestinal motility, and barrier function [80]. Many shortchain fatty acids (SCFAs) producing bacteria are decreased in IBD patients [51]. These include Faecalibacterium [81, 82], Odoribacter, Leuconostocaceae, Phascolarctobacterium, and Roseburia. Other metagenomic changes in patients with IBD include increase in the functions of auxotrophic and pathobiont bacteria, which include a decrease in amino acid biosynthesis and an increase in amino acid transporter genes [51]. There is an increase in sulphate-reducing bacteria such as Desulfovibrio [83], increased glutathione and riboflavin metabolism [51], and increased secretion of toxins along with an increase in bacterial genes related to virulence factors [84].

We also studied the interplay between butyrate concentration and butyrate-producing bacteria in fecal samples of UC patients compared to healthy controls. Fecal samples were collected from 14 control individuals (hemorrhoid patients only) and 26 UC patients (severe $n=12$, moderate $n=6$, remission $n=8$ ). Fluorescent in situ hybridization was employed in combination with flow cytometry to enumerate the Clostridium cluster population targeted by the $16 \mathrm{~S}$ rRNA gene probe. Major butyrate-producing species within this cluster were quantified to see if any change existed in control vs. UC patients with different disease activity. This observed change was further validated by qPCR. In addition to this, gas chromatography was used to evaluate the changes in concentration of major short-chain fatty acids (SCFAs), namely acetate, $n$-butyrate, and iso-butyrate. There was a significant decrease of Clostridium coccoides and Clostridium leptum clusters in fecal samples of UC patients. Furthermore, it was observed that some butyrate-producing members of the clostridial cluster, like Faecalibacterium prausnitzii $(p=0.0001)$ and Roseburia intestinalis were differentially present in patients with different disease activity. In addition, this study also showed decreased concentrations of fecal SCFAs, especially of $n$-butyrate, iso-butyrate, and acetate, in the fecal 
samples of UC patients. The observed decrease of predominant butyrate producers of Clostridial clusters correlated with the reduced SCFA levels in active UC patients. This was further confirmed by the restoration in the population of some butyrate producers with simultaneous increase in the level of SCFA in remission samples. The results of this study supported similar observations made in earlier studies [70, 85].

Therefore, alterations in the population of microbiota with respect to disease type and severity as described here may play a role as biomarkers that may help to predict disease predisposition, activity, severity, and responsiveness to therapy. One can predict the disease activity of UC by measuring the level of these species and concentration of SCFAs in stool samples and this method can be developed as a non-invasive marker for disease activity.

\section{Therapeutic applications of microbiota in IBD}

Therapeutics with microbiota in IBD include the use of probiotics that are defined as "live microorganisms, which when administered in adequate amounts confer a health benefit to the host [86]" and fecal microbiota transplantation. The beneficial effects on health by replacement of healthy bacterial flora are shown in Fig. 7.

There are specific requirements for probiotics before clinical use. They should be of human origin, genetically stable, and should be resistant to the acid, bile, and digestive enzymes in the GI tract. They should also have a safety record in human intervention trials. However, data on the use of probiotics are limited, and a strong consensus cannot be drawn from the current literature. In a recent meta-analysis [87] (which included only full text papers in English), there were 41 studies that were categorized on the basis of disease type and severity. There was marginal, non-statistically significant benefit with Bifidobacterium fermented milk versus placebo [88, 89] [RR: 2.7 (95 \% CI 0.47-15.33)] and VSL\#3 versus placebo [90-92] [RR: 1.88 (95\% CI 0.96-3.67)] in inducing remission in active UC. There was no benefit with $E$. coli Nissle 1917 versus standard treatment [93, 94] for preventing relapses in inactive UC, VSL\#3 versus placebo for preventing relapses in inactive UC [95]/ileo-anal pouch anastomosis [96-99]; preventing endoscopic recurrences in inactive $\mathrm{CD}$ with Lactobacillus rhamnosus $G G$ [100, 101] versus placebo and for preventing endoscopic recurrences in inactive CD with Lactobacillus johnsonii versus placebo [102]. The conclusions drawn from this meta-analysis were that the evidence from the current trials is weak and further welldesigned randomized controlled trials are required before probiotics can be recommended for treatment of IBD. In addition, probiotics have shown some evidence in induction of remission in cases of microscopic colitis [103].

Fecal microbiota transplantation (FMT) gained popularity with its excellent treatment efficacy in difficult-totreat patients with $C$. difficile infection [104, 105]. After 2013, data is emerging for its role in treating patients with IBD. A recent systemic review and meta-analysis of cohort

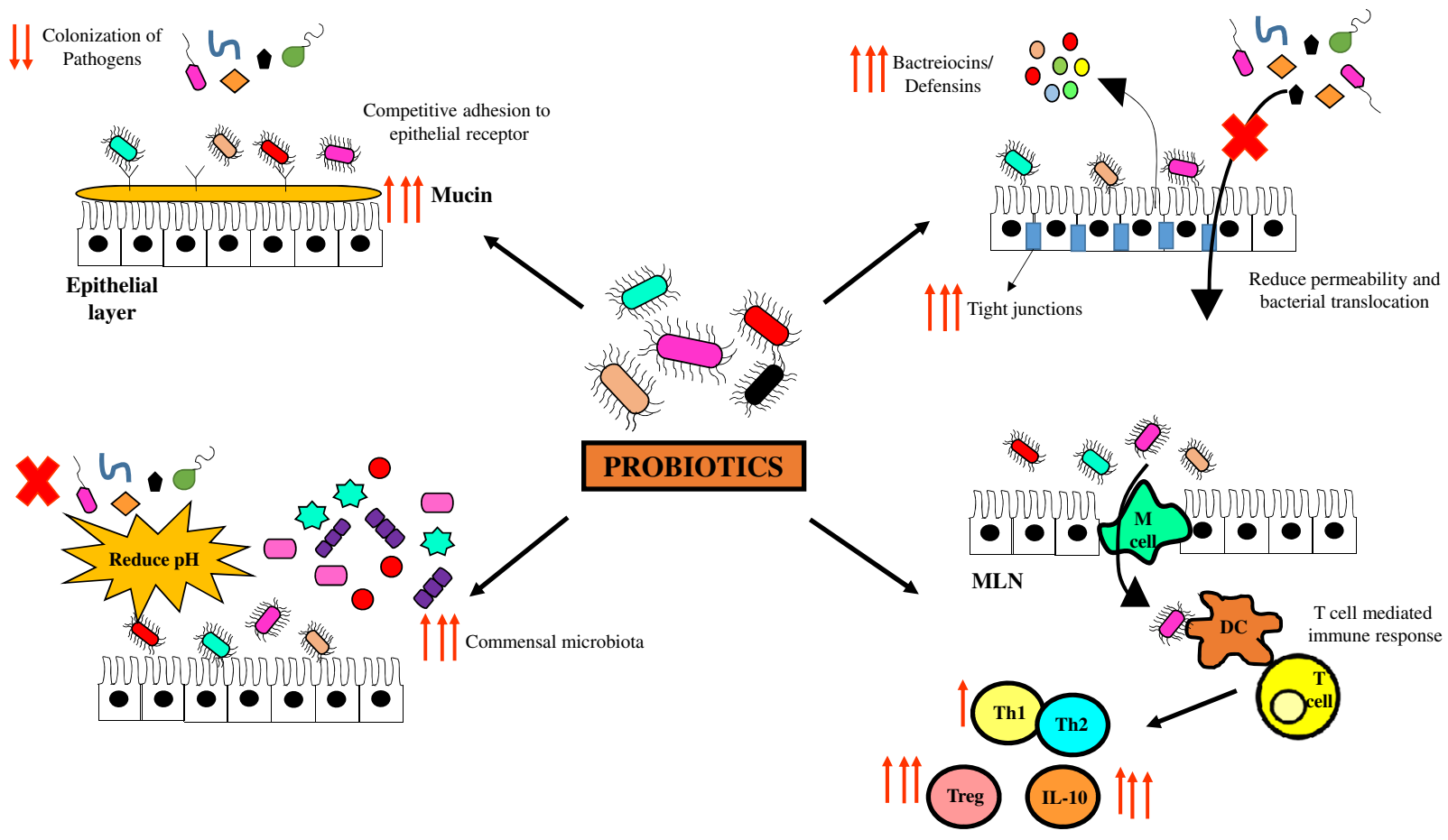

Fig. 7 Beneficial effects of probiotics on human gut 
studies clarified the role of FMT in IBD [106]. This metaanalysis included 18 studies (122 patients: $79 \mathrm{UC}, 39 \mathrm{CD}$, four IBD unclassified). The overall remission rate was $45 \%$ with FMT. However, in a meta-analysis of cohort studies, the pooled proportion of patients who achieved remission was $36.2 \%$. In the subgroup analyses, the proportion of UC patients who achieved remission was only $22 \%$ [107-111], and the proportion of CD patients who achieved clinical remission was $60.5 \%$ [112-115]. Most of the donors were first-degree relatives of these patients. FMT delivery methods were enemas, colonoscopic, nasogastric/nasojejunal, and gastroscopic installation. The FMT frequency was variable among the studies. FMT was well tolerated and no study reported any serious adverse events. Six studies also did microbiota analysis. There was a significant reduction in Proteobacteria and an increase in Bacteroidetes after FMT [110]. At the metagenomic level, there was an increase in butyrate-producing groups in responders as compared to non-responders. However, the data on FMT is still in its infancy. As per the current literature, it is a safe but variably efficacious treatment for IBD.

\section{Conclusions}

We studied the gut microbiome (both fecal and mucosal) in a varied spectrum of intestinal diseases ranging from acute and chronic infections to chronic autoimmune diseases like inflammatory bowel disease in a unique population which is endemic for infectious diseases and has an increasing burden of autoimmune diseases. We found the microbiome perturbations to be generally similar in infectious and autoimmune diseases. The population of useful bacteria such as lactobacilli was decreased in both amebiasis and ulcerative colitis while the population of pathogenetic bacteria were increased in both amebiasis and IBD. Although the microbiome would vary at the phylogenetic level, the alteration in functional composition was essentially similar in these diseases. Therefore the presence of inflammation would be the major engine for microbiome alterations in these diseases. These studies provide the platform for investigating microbiome diversity perturbations in diseases with varying etiology as well as a roadmap for therapeutic microbiome manipulation in a population which is unique as it displays a high prevalence of both enteric infections as well as gut autoimmune diseases.

\section{Compliance with ethical standards}

Conflict of interest The authors declare that they have no conflict of interest.

\section{References}

1. Whitman WB, Coleman DC, Wiebe WJ. Prokaryotes: the unseen majority. Proc Natl Acad Sci USA. 1998;95:6578-83.

2. Sonnenburg JL, Angenent LT, Gordon JI. Getting a grip on things: how do communities of bacterial symbionts become established in our intestine? Nat Immunol. 2004;5:569-73.

3. Xu J, Gordon JI. Honor thy symbionts. Proc Natl Acad Sci USA. 2003;100:10452-9.

4. Yatsunenko T, Rey FE, Manary MJ, Trehan I, et al. Human gut microbiome viewed across age and geography. Nature. 2012;486:222-7.

5. Mitsou EK, Kirtzalidou E, Oikonomou I, et al. Fecal microflora of Greek healthy neonates. Anaerobe. 2008;14:94-101.

6. Collado MC, Isolauri E, Laitinen K, et al. Effect of mother' $s$ weight on infant' s microbiota acquisition, composition, and activity during early infancy: a prospective follow-up study initiated in early pregnancy. Am J Clin Nutr. 2010;92:1023-30.

7. De Filippo C, Cavalieri D, Di Paola M, et al. Impact of diet in shaping gut microbiota revealed by a comparative study in children from Europe and rural Africa. PNAS. 2010;107:14691-6.

8. Turnbaugh PJ, Ridaura VK, Faith JJ, et al. The effect of diet on the human gut microbiome: a metagenomic analysis in humanized gnotobiotic mice. Sci Transl Med. 2009;1:6ra-14ra.

9. Goldsmith JR, Sartor RB. The role of diet on intestinal microbiota metabolism: downstream impacts on host immune function and health, and therapeutic implications. J Gastroenterol. 2014;49:785-98.

10. Hopkins MJ, Sharp R, Macfarlane GT. Age and disease related changes in intestinal bacterial populations assessed by cell culture, 16S rRNA abundance, and community cellular fatty acid profiles. Gut. 2001;48:198-205.

11. Khachatryan ZA, Ktsoyan ZA, Manukyan GP, et al. Predominant role of host genetics in controlling the composition of gut microbiota. PLoS One. 2008;3:e3064.

12. Dominguez-Bello MG, Blaser MJ, Ley RE, et al. Development of the human gastrointestinal microbiota and insights from highthroughput sequencing. Gastroenterology. 2011;140:1713-9.

13. Lozupone CA, Stombaugh JI, Gordon JI, et al. Diversity, stability and resilience of the human gut microbiota. Nature. 2012;489:220-30.

14. Claesson MJ, Cusack S, O' Sullivan O, et al. Composition, variability, and temporal stability of the intestinal microbiota of the elderly. Proc Natl Acad Sci USA. 2011;108(suppl 1):4586-91.

15. Maynard CL, Elson CO, Hatton RD, et al. Reciprocal interactions of the intestinal microbiota and immune system. Nature. 2012;489:231-2241.

16. Eckburg PB, Bik EM, Bernstein CN, et al. Diversity of the human intestinal microbial flora. Science. 2005;308:1635.

17. Hooper LV, Xu J, Falk PG, et al. A molecular sensor that allows a gut commensal to control its nutrient foundation in a competitive ecosystem. Proc Natl Acad Sci USA. 1999;96:9833-8.

18. Kanauchi O, Fujiyama Y, Mitsuyama K, et al. Increased growth of Bifidobacterium and Eubacterium by germinated barley foodstuff, accompanied by enhanced butyrate production in healthy volunteers. Int J Mol Med. 1999;3:175-9.

19. Simmering R, Kleessen B, Blaut M. Quantification of the flavonoid-degrading bacterium Eubacterium ramulus in human fecal samples with a species-specific oligonucleotide hybridization probe. Appl Env Microbiol. 1999;65:3705-9.

20. Flint HJ. Polysaccharide breakdown by anaerobic microorganisms inhabiting the mammalian gut. Adv Appl Microbiol. 2004;56:89-120. 
21. Hoskins LC. Mucin degradation in the human gastrointestinal tract and its significance to enteric microbial ecology. Eur $\mathbf{J}$ Gastroenterol Hepatol. 1992;5:203-13.

22. Lebeer S, Vanderleyden J, De Keersmaecker SCJ. Genes and molecules of Lactobacilli supporting probiotic action. Microbiol Mol Biol Rev. 2008;72:728-64.

23. Van Neil CW, Feudtner C, Garrison MM, et al. Lactobacillus therapy for acute infectious diarrhea in children: a meta analysis. Pediatrics. 2002;109:678-84.

24. Fukuda S, Toh H, Hasel K, et al. Bifidobacteria can protect from enteropathogenic infection through production of acetate. Nature. 2011;469:543-7.

25. Leitch EC, Walker AW, Duncan SH, et al. Selective colonization of insoluble substrates by human faecal bacteria. Env Microbiol. 2007;9:667-79.

26. Deplancke B, Hristova KR, Oakley HA, et al. Molecular ecological analysis of the succession and diversity of sulphate-reducing bacteria in mouse gastrointestinal tract. Appl Env Microbiol. 2000;66:2166-74.

27. Goldstein EJC, Citron DM, Peraino VA, et al. Desulfovibrio desulfuricans bacteremia and review of human Desulfovibrio infections. J Clin Microbiol. 2003;41:2752-4.

28. Guarner F. Enteric flora in health and disease. Digestion. 2006;73(suppl 1):5-12.

29. O'Hara AM, Shanahan F. The gut flora as a forgotten organ. EMBO Rep. 2006;7:688-93.

30. Walsh JA. Problems in recognition and diagnosis of amebiasis: estimation of the global magnitude of morbidity and mortality. Rev Infect Dis. 1996;8:228-38.

31. Sharma MP, Ahuja V. Amoebic liver abscess. J Indian Acad Clin Med. 2003;4:107-11.

32. Katzenstein D, Rickerson V, Braude A. New concepts of amebic liver abscess derived from hepatic imaging, serodiagnosis, and hepatic enzymes in 67 consecutive cases in San Diego. Medicine (Baltimore). 1982;68:237-46.

33. Haque R, Huston CD, Hughes M, et al. Amoebiasis. N Engl J Med. 2003;348:1565-73.

34. Simon GL, Gorbach SL. Intestinal flora in health and disease. Gastroenterology. 1984;86:174-93.

35. Nakamura M. Nutrition and physiology of Entamoeba histolytica. Bacteriol Rev. 1953;17:189-212.

36. Bracha R, Kobiler D, Mirelman D. Attachment and ingestion of bacteria by trophozoite of Entamoeba histolytica. Am J Hyg. 1982;5:371-405.

37. Mirelman D. Ameba-bacterium relationship in amoebiasis. Microbiol Rev. 1987;51:272-84.

38. Rani R, Murthy RS, Bhattacharya S, et al. Changes in bacterial profile during amebiasis: demonstration of anaerobic bacteria in ALA pus samples. Am J Trop Med Hyg. 2006;75:880-5.

39. Ralph ED, Kirby WM. Bioassay of metronidazole with either anaerobic or aerobic. J Infect Dis. 1975;132:587-91.

40. Lofmark S, Fang H, Hedberg M, et al. Inducible metronidazole resistance and nim genes in clinical Bacteroides fragilis group isolates. Antimicrob Agents Chemother. 2005;49:1253-6.

41. Reysset G, Haggoud A, Sebald M. Genetics of resistance of Bacteroides species to 5-nitroimidazole. Clin Infect Dis. 1993;16:S401-3.

42. Stubbs SLJ, Brazier JS, Talbot PR, et al. PCR-restriction fragment length polymorphism analysis for identification of Bacteroides spp. and characterization of nitroimidazole resistance genes. J Clin Microbiol. 2000;38:3209-13.

43. Theron MM, Rensburg MNJV, Chalkley LJ, et al. Nitroimidazole resistance genes ( nim $b$ ) in anaerobic Gram-positive cocci (previously Peptostreptococcus spp.). J Antimicrob Chemother. 2004;54:240-2.
44. Strober W, Fuss I, Mannon P. The fundamental basis of inflammatory bowel disease. J Clin Invest. 2007;117:514-21.

45. Pirzer U, Schonhaar A, Fleischer B, et al. Reactivity of infiltrating $\mathrm{T}$ lymphocytes with microbial antigens in Crohn' s disease. Lancet. 1991;338:1238-9.

46. Macpherson A, Khoo UY, Forgacs I, et al. Mucosal antibodies in inflammatory bowel disease are directed against intestinal bacteria. Gut. 1996;38:365-75.

47. Prantera C, Lochs H, Grimaldi M, et al. Retic study group (Rifaximin-Eir Treatment in Crohn's Disease). Rifaximin-extended intestinal release induces remission in patients with moderately active Crohn's disease. Gastroenterology. 2012;142:473-81.

48. Sheehan D, Moran C, Shanahan F. The microbiota in inflammatory bowel disease. J Gastroenterol. 2015;50:495-507.

49. Frank DN, Robertson CE, Hamm CM, et al. Disease phenotype and genotype are associated with shifts in intestinal-associated microbiota in inflammatory bowel diseases. Inflamm Bowel Dis. 2011;17:179-84.

50. Morgan XC, Tickle TL, Sokol H, et al. Dysfunction of the intestinal microbiome in inflammatory bowel disease and treatment. Genome Biol. 2012;13:R79.

51. Ahuja V. Inventory of a reservoir: friends \& foes. Indian J Med Res. 2015;142:4-6.

52. Ott SJ, Kuhbacher T, Musfeldt M, et al. Fungi and inflammatory bowel diseases: alterations of composition and diversity. Scand $\mathbf{J}$ Gastroenterol. 2008;43:831-41.

53. Trojanowska D, Zwolinska-Wcislo M, Tokarczyk M, et al. The role of Candida in inflammatory bowel disease. Estimation of transmission of $C$. albicans fungi in gastrointestinal tract based on genetic affinity between strains. Med Sci Monit. 2010;16:451-7.

54. Lupp C, Robertson ML, Wickham ME, et al. Host-mediated inflammation disrupts the intestinal microbiota and promotes the overgrowth of Enterobacteriaceae. Cell Host Microbe. 2007;2:119-29.

55. Darfeuille-Michaud A, Boudeau J, Bulois P, et al. High prevalence of adherent-invasive Escherichia coli associated with ileal mucosa in Crohn's disease. Gastroenterology. 2004;127:412-21.

56. Sokol H, Lepage P, Seksik P, et al. Temperature gradient gel electrophoresis of fecal 16S rRNA reveals active Escherichia coli in the microbiota of patients with ulcerative colitis. J Clin Microbiol. 2006;44:3172-7.

57. Ohkusa T, Sato N, Ogihara T, et al. Fusobacterium varium localized in the colonic mucosa of patients with ulcerative colitis stimulates species-specific antibody. J Gastroenterol Hepatol. 2002; 17:849-53.

58. Ohkusa T, Okayasu I, Ogihara T, et al. Induction of experimental ulcerative colitis by Fusobacterium varium isolated from colonic mucosa of patients with ulcerative colitis. Gut. 2003;52:79-83.

59. Llopis M, Antolin M, Carol M, et al. Lactobacillus casei downregulates commensals' inflammatory signals in Crohn's disease mucosa. Inflamm Bowel Dis. 2009;15:275-83.

60. Willing B, Halfvarson J, Dicksved J, et al. Twin studies reveal specific imbalances in the mucosa-associated microbiota of patients with ileal Crohn's disease. Inflamm Bowel Dis. 2009;15:653-60.

61. Sokol H, Pigneur B, Watterlot L, et al. Faecalibacterium prausnitzii is an anti-inflammatory commensal bacterium identified by gut microbiota analysis of Crohn disease patients. Proc Natl Acad Sci USA. 2008;105:16731-6.

62. Verma R, Verma AK, Ahuja V, et al. Real-time analysis of mucosal flora in patients with inflammatory bowel disease in India. J Clin Microbiol. 2010;48:4279-82. 
63. Conte MP, Schippa S, Zamboni I, Penta M, Chiarini F, Seganti L, Osborn J, Falconieri P, Borrelli O, Cucchiara S. Gut-associated bacterial microbiota in paediatric patients with inflammatory bowel disease. Gut. 2006;55(12):1760-7.

64. Ott SJ, Plamondon S, Hart A, Begun A, Rehman A, Kamm MA, Schreiber S. Dynamics of the mucosa-associated flora in ulcerative colitis patients during remission and clinical relapse. J Clin Microbiol. 2008;46(10):3510-3.

65. Zhang MB, Liu Y, Zhang H, et al. Structural shifts of mucosaassociated lactobacilli and Clostridium leptum subgroup in patients with ulcerative colitis. J Clin Microbiol. 2007;45:496-500.

66. Marteau P. Bacterial flora in inflammatory bowel disease. Dig Dis. 2009;27(Suppl. 1):99-103.

67. Sokol H, Lay C, Seksik P, et al. Analysis of bacterial bowel communities of IBD patients: what has it revealed? Inflamm Bowel Dis. 2008;14:858-67.

68. van de Merwe JP, Mol GJ. A possible role of Eubacterium and Peptostreptococcus species in the aetiology of Crohn's disease. Antonie Van Leeuwenhoek. 1980;46:587-93.

69. Takaishi H, Matsuki $T$, Nakazawa $A$, et al. Imbalance in intestinal microflora constitution could be involved in the pathogenesis of inflammatory bowel disease. Int J Med Microbiol. 2008;298:463-72.

70. Andoh A, Imaeda H, Aomatsu T, et al. Comparison of the fecal microbiota profiles between ulcerative colitis and Crohn's disease using terminal restriction fragment length polymorphism analysis. J Gastroenterol. 2011;46:479-86.

71. Vander Wiel JA, Korstanie JA, Winkler KC. The faecal flora in ulcerative colitis. J Med Microbiol. 1975;8:491-501.

72. Huda-Faujan N, Abdulamir AS, Fatimah AB, et al. The impact of the level of the intestinal short-chain fatty acids in inflammatory bowel disease patients versus healthy subjects. Open Biochem J. 2010;4:1-14.

73. Cummings JH, Macfarlane GT, Englyst HN. Prebiotic digestion and fermentation. Am J Clinic Nutr. 2001;73:415S-20S.

74. Rhodes JM. Colonic mucus and ulcerative colitis. Gut. 1997;40:807-8.

75. Buisine MP, Desreumaux P, Leteurtre E, et al. Mucin gene expression in intestinal epithelial cells in Crohn's disease. Gut. 2001;49:544-51.

76. Scheppach W. Effects of short-chain fatty acids on gut morphology and function. Gut. 1994;35:S35-8.

77. Ahmad MS, Krishnan S, Ramakrishna BS, et al. Butyrate and glucose metabolism by colonocytes in experimental colitis in mice. Gut. 2000;46:493-9.

78. Smith PM, Howitt MR, Panikov N, et al. The microbial metabolites, short-chain fatty acids, regulate colonic Treg cell homeostasis. Science. 2013;341:569-73.

79. Segain JP, Raingeard de la Blétière D, Bourreille A, et al. Butyrate inhibits inflammatory responses through NFkappaB inhibition: implications for Crohn's disease. Gut. 2000;47:397-403.

80. Peng L, Li ZR, Green RS, et al. Butyrate enhances the intestinal barrier by facilitating tight junction assembly via activation of AMP-activated protein kinase in Caco- 2 cell monolayers. J Nutr. 2009; 139:1619-25.

81. Frank DN, St Amand AL, Feldman RA, et al. Molecular phylogenetic characterization of microbial community imbalances in human inflammatory bowel diseases. Proc Natl Acad Sci USA. 2007;104:13780-5.

82. Sokol H, Seksik P, Furet JP, et al. Low counts of Faecalibacterium prausnitzii in colitis microbiota. Inflamm Bowel Dis. 2009;15:1183-9.

83. Rowan F, Docherty NG, Murphy M, et al. Desulfovibrio bacterial species are increased in ulcerative colitis. Dis Colon Rectum. 2010;53:1530-6.
84. Erickson AR, Cantarel BL, Lamendella R, et al. Integrated metagenomics/metaproteomics reveals human host-microbiota signatures of Crohn's disease. PLoS One. 2012;7:e49138.

85. Sokol H, Seksik P, Rigottier-Gois L, et al. Specificities of the fecal microbiota in inflammatory bowel disease. Inflamm Bowel Dis. 2006; 12:106-11.

86. Food and Agriculture Organization of the United Nations, WHO. Joint FAO/WHO expert consultation on evaluation of health and nutritional properties of probiotics in food including powder milk with live lactic acid bacteria. Cordoba: 2001 Oct [online].

87. Jonkers D, Penders J, Masclee A, et al. Probiotics in the management of inflammatory bowel disease: a systematic review of intervention studies in adult patients. Drugs. 2012;16(72):803-23.

88. Ishikawa H, Akedo I, Umesaki Y, et al. Randomized controlled trial of the effect of Bifidobacteria-fermented milk on ulcerative colitis. J Am Coll Nutr. 2003;22:56-63.

89. Kato K, Mizuno S, Umesaki Y, et al. Randomized placebocontrolled trial assessing the effect of Bifidobacteria-fermented milk on active ulcerative colitis. Aliment Pharmacol Ther. 2004;20:1133-41.

90. Bibiloni R, Fedorak RN, Tannock GW, et al. VSL\#3 probioticmixture induces remission in patients with active ulcerative colitis. Am J Gastroenterol. 2005;100:1539-46.

91. Sood A, Midha V, Makharia GK, et al. The probiotic preparation, VSL\#3 induces remission in patients with mild-to-moderately active ulcerative colitis. Clin Gastroenterol Hepatol. 2009;7:1202-9 (9 e1.).

92. Tursi A, Brandimarte G, Papa A, et al. Treatment of relapsing mild-to-moderate ulcerative colitis with the probiotic VSL\#3 as adjunctive to a standard pharmaceutical treatment: a doubleblind, randomized, placebo controlled study. Am J Gastroenterol. 2010;105:2218-27.

93. Kruis W, Schutz E, Fric P, et al. Double-blind comparison of an oral Escherichia coli preparation and mesalazine in maintaining remission of ulcerative colitis. Aliment Pharmacol Ther. 1997;11:853-8.

94. Kruis W, Fric P, Pokrotnieks J, et al. Maintaining remission of ulcerative colitis with the probiotic Escherichia coli Nissle 1917 is as effective as with standard mesalazine. Gut. 2004;53:1617-23.

95. Venturi A, Gionchetti P, Rizzello F, et al. Impact on the composition of the faecal flora by a new probiotic preparation: preliminary data on maintenance treatment of patients with ulcerative colitis. Aliment Pharmacol Ther. 1999;13:1103-8.

96. Gionchetti P, Rizzello F, Venturi A, et al. Oral bacteriotherapy as maintenance treatment in patients with chronic pouchitis: a double-blind, placebo-controlled trial. Gastroenterology. 2000;119:305-9.

97. Gionchetti P, Rizzello F, Helwig U, et al. Prophylaxis of pouchitis onset with probiotic therapy: a double-blind, placebocontrolled trial. Gastroenterology. 2003;124:1202-9.

98. Mimura T, Rizzello F, Helwig U, et al. Once daily high dose probiotic therapy (VSL\#3) for maintaining remission in recurrent or refractory pouchitis. Gut. 2004;53:108-14.

99. Shen B, Brzezinski A, Fazio VW, et al. Maintenance therapy with a probiotic in antibiotic-dependent pouchitis: experience in clinical practice. Aliment Pharmacol Ther. 2005;22:721-8.

100. Prantera C, Scribano ML, Falasco G, et al. Ineffectiveness of probiotics in preventing recurrence after curative resection for Crohn's disease: a randomised controlled trial with Lactobacillus GG. Gut. 2002;51:405-9.

101. Schultz M, Timmer A, Herfarth HH, et al. Lactobacillus GG in inducing and maintaining remission of Crohn's disease. BMC Gastroenterol. 2004;4:5.

102. Marteau P, Lemann M, Seksik P, et al. Ineffectiveness of Lactobacillus johnsonii LA1 for prophylaxis of post-operative 
recurrence in Crohn's disease: a randomised, double blind, placebo controlled GETAID trial. Gut. 2006;55:842-7.

103. Rohatgi S, Ahuja V, Makharia GK, Rai T, Das P, Dattagupta S, Mishra V, Garg SK. VSL\#3 induces and maintains short-term clinical response in patients with active microscopic colitis: a two-phase randomised clinical trial. BMJ Open Gastroenterol. 2015;2(1):e000018.

104. Kassam Z, Lee CH, Yuan Y, et al. Fecal microbiota transplantation for Clostridium difficile infection: systematic review and meta-analysis. Am J Gastroenterol. 2013;108:500-8.

105. Cammarota G, Ianiro G, Gasbarrini A. Fecal microbiota transplantation for the treatment of Clostridium difficile infection: a systematic review. J Clin Gastroenterol. 2014;48:693-702.

106. Colman RJ, Rubin DT. Fecal microbiota transplantation as therapy for inflammatory bowel disease: a systematic review and meta-analysis. J Crohns Colitis. 2014;8:1569-81.

107. Angelberger S, Reinisch W, Makristathis A, et al. Temporal bacterial community dynamics vary among ulcerative colitis patients after fecal microbiota transplantation. Am J Gastroenterol. 2013;108:1620-30.

108. Damman C, Brittnacher M, Hayden H, et al. Single colonoscopically administered fecal microbiota transplant for ulcerative colitis - a pilot study to determine therapeutic benefit and graft stability. Gastroenterology. 2014;146:S-460.
109. Kump PK, Grochenig HP, Lackner S, et al. Alteration of intestinal dysbiosis by fecal microbiota transplantation does not induce remission in patients with chronic active ulcerative colitis. Inflamm Bowel Dis. 2013;19:2155-65.

110. Kunde S, Pham A, Bonczyk S, et al. Safety, tolerability, and clinical response after fecal transplantation in children and young adults with ulcerative colitis. J Pediatr Gastroenterol Nutr. 2013;56:597-601.

111. Landy J, Al-Hassi HO, Mann ER, et al. Tu1985 A prospective controlled pilot study of fecal microbiota transplantation for chronic refractory pouchitis. Gastroenterology. 2013;144:S-897.

112. Suskind D, Wahbeh G, Vendetoulli H, et al. Fecal microbial transplant in pediatric Crohn's disease. Gastroenterology. 2014;146:S-834.

113. Vaughn BP, Gevers D, Ting A, et al. Fecal microbiota transplantation induces early improvement in symptoms in patients with active Crohn's disease. Gastroenterology. 2014;146:S591-2.

114. Vermeire S, Joossens M, Verbeke K, et al. Sa1922 Pilot study on the safety and efficacy of faecal microbiota transplantation in refractory Crohn's disease. Gastroenterology. 2012;142:S-360.

115. Zhang F-M, Wang H-G, Wang M, et al. Standard fecal microbiota transplantation through mid-gut is an effective therapy of refractory Crohn's disease. J Gastroenterol Hepatol. 2013;28:9. 